\title{
Biomarkers of food intake for cocoa and liquorice (products): a systematic review
}

Charlotte C. J. R. Michielsen ${ }^{1}$, Enrique Almanza-Aguilera ${ }^{2,3}$, Elske M. Brouwer-Brolsma', Mireia Urpi-Sarda 2,3 and Lydia A. Afman ${ }^{1 *}$

\begin{abstract}
Background: To unravel true links between diet and health, it is important that dietary exposure is accurately measured. Currently, mainly self-reporting methods (e.g. food frequency questionnaires and 24-h recalls) are used to assess food intake in epidemiological studies. However, these traditional instruments are subjective measures and contain well-known biases. Especially, estimating the intake of the group of confectionary products, such as products containing cocoa and liquorice, remains a challenge. The use biomarkers of food intake (BFIs) may provide a more objective measurement. However, an overview of current candidate biomarkers and their validity is missing for both cocoa- and liquorice-containing foods.
\end{abstract}

Objective: The purpose of the current study was to (1) identify currently described candidate BFls for cocoa (products) and liquorice, (2) to evaluate the validity of these identified candidate BFls and (3) to address further validation and/or identification work to be done.

Methods: This systematic review was based on a comprehensive literature search of three databases (PubMed, Scopus and ISI web of Science), to identify candidate BFIs. Via a second search step in the Human Metabolome Database (HMDB), the Food Database (FooDB) and Phenol-Explorer, the specificity of the candidate BFIs was evaluated, followed by an evaluation of the validity of the specific candidate BFIs, via pre-defined criteria.

Results: In total, 37 papers were included for cocoa and 8 papers for liquorice. For cocoa, 164 unique candidate BFIs were obtained, and for liquorice, four were identified in total. Despite the high number of identified BFIs for cocoa, none of the metabolites was specific. Therefore, the validity of these compounds was not further examined. For liquorice intake, 18-glycyrrhetinic acid (18-GA) was found to have the highest assumed validity.

Conclusions: For cocoa, specific BFls were missing, mainly because the individual BFIs were also found in foods having a similar composition, such as tea (polyphenols) or coffee (caffeine). However, a combination of individual BFIs might lead to discriminating profiles between cocoa (products) and foods with a similar composition. Therefore, studies directly comparing the consumption of cocoa to these similar products are needed, enabling efforts to find a unique profile per product. For liquorice, we identified 18-GA as a promising BFl; however, important information on its validity is missing; thus, more research is necessary. Our findings indicate a need for more studies to determine acceptable BFIs for both cocoa and liquorice.

Keywords: Licorice, Liquorice, Cocoa, Cacao, Chocolate, Metabolites, Metabolomics, Biomarkers

\footnotetext{
* Correspondence: Iydia.afman@wur.nl

${ }^{1}$ Division of Human Nutrition and Health, Wageningen University and

Research Centre, Stippeneng 4, 6708 WE Wageningen, The Netherlands

Full list of author information is available at the end of the article
}

(c) The Author(s). 2018 Open Access This article is distributed under the terms of the Creative Commons Attribution 4.0 International License (http://creativecommons.org/licenses/by/4.0/), which permits unrestricted use, distribution, and reproduction in any medium, provided you give appropriate credit to the original author(s) and the source, provide a link to the Creative Commons license, and indicate if changes were made. The Creative Commons Public Domain Dedication waiver (http://creativecommons.org/publicdomain/zero/1.0/) applies to the data made available in this article, unless otherwise stated. 


\section{Background}

Several epidemiological studies have observed relationships between habitual intake of cocoa (products) and liquorice and health. Beneficial effects associated to the consumption of cocoa and cocoa product intake include a positive association with flow-mediated vasodilatation and inverse associations with blood pressure, serum insulin, HOMA-IR, calcified atherosclerotic plaques in the coronary arteries, incident cardiovascular diseases, cardiac mortality, cardiovascular mortality and all-cause mortality [1-6]. Liquorice has been widely exploited for medicinal purposes including its use as remedy in case of sore throat or cough $[7,8]$. However, even though beneficial effects have been reported for both the consumption of cocoa- and liquorice-containing products, many of these products are often energy-dense foods, high in sugar and fats. Therefore, intake of these products in high amounts is not recommended, as it is associated with obesity and related diseases $[9,10]$. Also, contraindications for the use of liquorice in large amounts have been reported in specific conditions, especially during pregnancy and in patients with hypertension, hypokalaemia or hepatic or kidney failure $[11,12]$.

To unravel true links between diet and health in epidemiology studies, it is essential to accurately assess dietary exposure. Currently, the use of self-reporting methods, such as 24-h dietary recalls, food diaries, and food-frequency questionnaires are the most frequently used instruments to assess food exposure in epidemiological studies. However, these conventional methods are subjective measures; they contain well-known biases, such as reporting and recall biases [13, 14], and do not take into account individual characteristics and differential metabolic responses to the intake of different food components and food bioactives. Therefore, the observed relationships between nutrition and health could have been affected, thereby possibly leading to inconsistencies in the field of nutritional research. In particular, accurate estimation of the intake of cocoa- and liquorice-containing products is difficult. This is partly due to the fact that the moments on which these types of foods are consumed are often not planned. Furthermore, people have difficulties determining accurate portion sizes, they might underreport their intake of these types of food as they are believed to be unhealthy, and they might not be able to recall all the foods that contain these types of compounds. Hence, there is an urgent need for more accurate measurements of food intake, especially for cocoa- and liquorice-containing foods. As a result of the application of metabolomics in the nutrition field, candidate biomarkers of food intake (BFIs) are increasingly described in the literature [15]. BFIs are objective measures of actual food intake and as such could be used in conjunction with the conventional methods, to improve the quality of dietary assessment in nutritional science and to assist in examining true associations between nutrition and health [16]. In the literature, many different biomarker classification schemes exist $[17,18]$. In this manuscript, we defined BFIs according to the definition proposed by Gao et al. [18]. In short, we included all biomarkers described in the literature after the intake of cocoa products, liquorice products or their components that can be used to estimate recent or average intakes of these entities. Since cocoa and cocoa products are consumed all over the world, the number of potential BFIs for cocoa (products) is rapidly rising in the literature. Liquorice is also widely consumed, especially in Europe, and can be used as an ingredient in different food products. However, potential BFIs for the consumption of liquorice (products) are less well explored [19]. Currently, three approaches are used for BFI identification, namely, (1) acute or chronic intervention studies where metabolic profiles are examined after a specific load of the food of interest, (2) dietary pattern studies where metabolic profiles are examined after subjects adhere to a certain dietary pattern and (3) observational studies where metabolic profiles are compared between consumers and non-consumers of a specific food of interest [20, 21]. To examine the validity of a candidate BFI, it is important that the BFI has been evaluated using all of these approaches. An overview of already identified candidate BFIs for cocoa (products) and liquorice, as well as an evaluation of their validity, is needed to identify known and accepted BFIs, as well as to identify what information is still missing and requires further investigation. Hence, the aims of this systematic review were (1) to identify currently described candidate BFIs for cocoa (products) and liquorice in the literature, (2) to evaluate the validity of these identified candidate BFIs and (3) to address further identification and/or validation work to be done. This systematic review is performed in the frame of the FoodBAll (Food Biomarkers Alliance) project under the Joint Programming Initiative-A Healthy Diet for a Healthy Life (JPI-HDHL) (http://www.foodmetabolome.org/).

\section{Materials and methods}

\section{Identification of biomarkers}

In order to identify papers on BFIs for both cocoa and liquorice, we carried out an extensive literature search following the Biomarker of Food Intake Reviews (BFIRev) methodology proposed previously [22]. In short, for this systematic review, all elements of the PRISMA statement [23] relevant for a literature search on biomarkers were used. Original papers and reviews were searched in Scopus, PubMed and ISI Web of Knowledge, using the grouped search terms (biomarker" OR marker* OR metabolite* OR biokinetics OR kinetic* 
OR biotransformation) AND (human* OR men OR women OR patient* OR volunteer* OR participant*) AND (trial OR experiment OR study OR intervention) AND (urine OR plasma OR serum OR blood OR excretion) AND (intake OR meal OR diet OR ingestion OR consumption OR eating OR drink* OR administration). For the BFI for cocoa, AND (cocoa* OR chocolate* OR cacao* OR Theobroma) was added, and for the BFI for liquorice, AND (liquorice OR liquorice) was added. Three independent reviewers (CCJRM, EAA and EMB-B) selected the papers in a process outlined in Fig. 1. Only English papers were included, and no restriction was applied for the publication dates (searches for cocoa and liquorice biomarkers were done up to October 2016 and March 2017, respectively). Papers describing the effect on physiology, bio stability and/or drug metabolism were excluded. Furthermore, we excluded papers that examined chocolate products without cocoa (for example white chocolate) and liquorice products without liquorice root extract (for example red liquorice). Animal studies were also excluded, since it remains to be determined whether animal models are valid models for examining the absorption, distribution, metabolism and excretion of compounds in humans [24]. Initially, only titles and abstracts were screened to determine if they satisfied the selection criteria. Any disagreements between the reviewers were resolved through consultation. Next, full-text papers were retrieved for the selected titles. Additional papers were identified from reference lists of the retrieved papers and from the selected book chapters or reviews, called hand searches. Again, three independent reviewers (CCJRM, EAA and EMB-B) assessed the obtained papers to ensure that they were in agreement with the inclusion criteria. A data collection form was designed to streamline the process of extracting relevant information from the selected studies. This form contained the following items: dietary factor, dose of intervention, study design, number of subjects, analytical method, sample type, discriminating metabolites (BFIs), notes and primary reference(s).

\section{Specificity evaluation of the identified biomarkers}

To evaluate the apparent specificity of each of the identified candidate BFI, a second search step consisting of two parts was performed. Firstly, the compound databases HMDB (http://www.hmdb.ca/), FooDB (http://foodb.ca/) and Phenol-Explorer (http://phenol-explorer.eu/) were used to screen the identified biomarkers of food intake. If a compound was found to be present in non-cocoa- or non-liquorice-related foods, it was removed from the selection. Secondly, an additional search was performed for the remaining selection, using combinations of the grouped search terms ("the name and synonyms of the compound") AND (biomarker" OR marker* OR metabolite* OR biokinetics OR biotransformation) AND (trial OR experiment OR study OR intervention) AND (human*

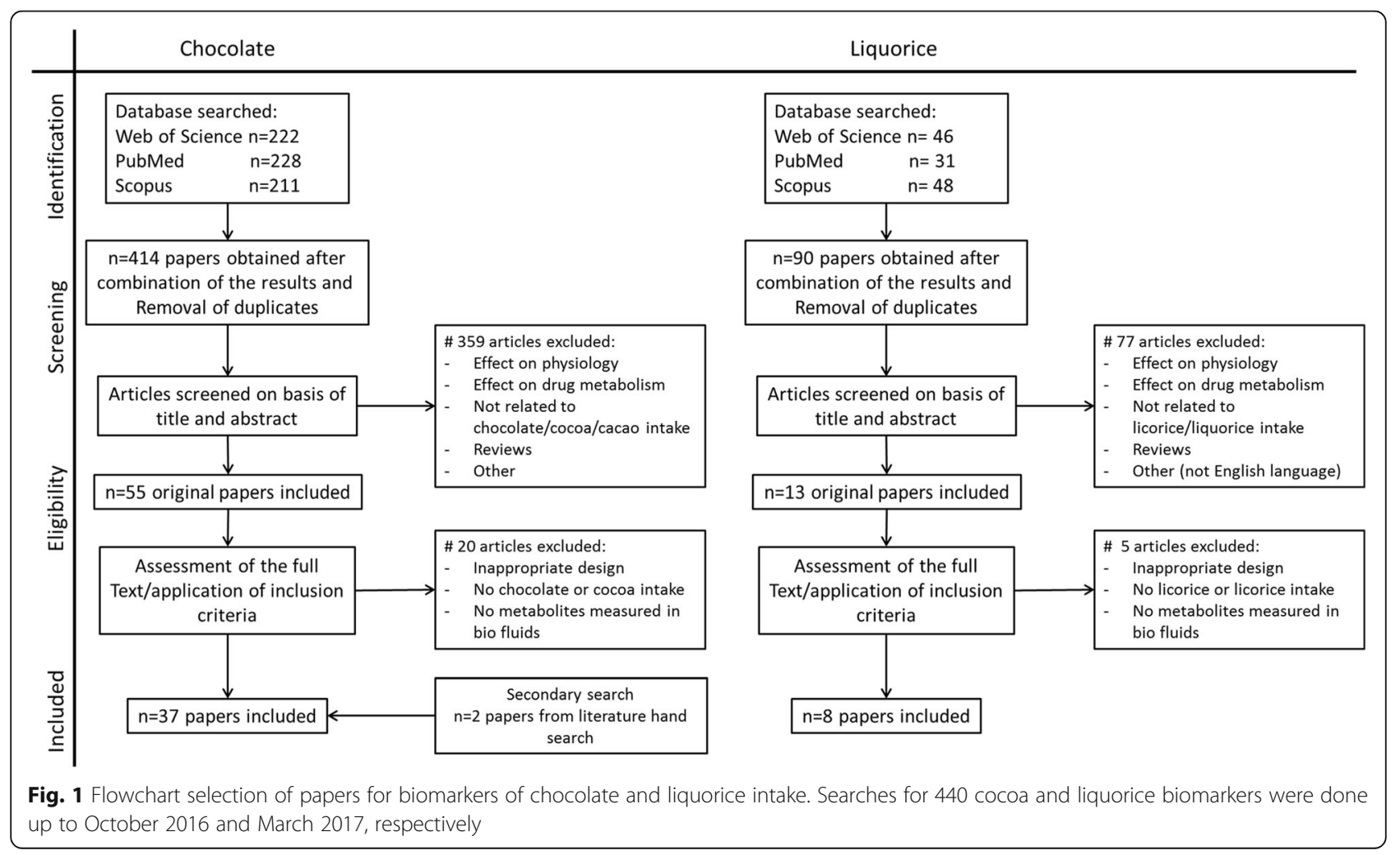


OR men OR women OR patient* OR volunteer* OR participant*) AND (urine OR plasma OR serum OR blood OR excretion) AND (intake OR meal OR diet OR ingestion OR consumption OR eating OR drink" OR administration) in any of the listed databases above or Google Scholar. If the compound was found to be present in non-cocoa- or non-liquorice-related foods in this second search, it was removed from the selection.

\section{Validity of the identified biomarkers}

For the final selection of identified BFIs, the validity was assessed. For this, we have used the method proposed by Dragsted et al. [25]. An elaborate explanation on how the validity was assessed, including the pre-defined criteria; how the validity questions were constructed; and a discussion on the strengths and weaknesses of this method can be found in the paper of Dragsted and colleagues [25]. In short, the validity was assessed via answering nine questions, where possible answers were Yes $(\mathrm{Y})$, No $(\mathrm{N})$ or unknown/uncertain $(\mathrm{U})$ where appropriate. If $\mathrm{Y}$ was answered to a question, this would increase the assumed validity of that specific biomarker. The questions taken into consideration were related to current knowledge about biological, analytical and nutritional aspects of the methodology and are based on a thorough search of previous literature. The questions were as follows: (1) Is the marker compound plausible as a specific BFI for the food or food group (chemical/biological plausibility)? (2) Is there a dose-response relationship at relevant intake levels of the targeted food (quantitative aspect)? (3) Is the single-meal timeresponse relationship described adequately to make a wise choice of sample type and time window (singledose kinetics)? (4) Is the biomarker kinetics for repeated intakes of the food/food group described adequately providing the frequency of sampling needed to assess habitual intake (e.g. the cumulative aspects: does the biomarker accumulate in the body over time after repeated intakes?)? (5) Has the marker been shown to be robust after intake of complex meals reflecting dietary habits of the targeted population (robustness)? (6) Has the marker been shown to compare well with other markers or questionnaire data for the same food/food group (reliability)? (7) Is the marker chemically and biologically stable during bio specimen collection and storage, making measurements reliable and feasible? (8) Are analytical variability (CV\%), accuracy, sensitivity and specificity known as adequate for at least one reported analytical method? (9) Has the analysis been successfully reproduced in another laboratory (reproducibility)? In the end, the number of times a $\mathrm{Y}$ was given per biomarker was added, in order to get insight in the validity of a selected biomarker. The higher this number, the more is known about the compound, the higher its assumed validity. This score will therefore reflect the current level of validity of that particular compound and pinpoints what additional research is needed to increase the validity of that particular compound.

\section{Results \\ Candidate BFIs, identification \\ Cocoa (products) BFIs}

A total of 414 potentially relevant papers were identified from searches in PubMed, Web of Science and Scopus. After a first screening of the title and abstract, 55 papers were collected as full text and assessed for further inclusion. Then, 20 papers were excluded due to either inappropriate study designs (e.g. animal studies), unreported cocoa or chocolate intakes or unreported metabolites/markers. Finally, two additional papers were identified via hand searches and added to the list, which lead to a total inclusion of 37 papers (Fig. 1). Intervention studies $(n=34)$ were the most frequently employed methods to determine candidate BFIs for cocoa (products) (Additional file 1: Table S1). In these intervention studies, the participants consumed the following: cocoa-based beverages $(n=20)$, chocolate $(n=8)$, a mixture of chocolate and cocoa beverages $(n=2)$, cocoa-based nut cream and/or polyphenol capsules $(n=2)$ and cocoa extract as part of a ready-to-eat meal $(n=1)$. Of the 34 intervention studies, there were 16 acute crossover studies, 12 acute single-dose studies, 3 crossover intervention studies (ranging from 4 to 6 weeks), 3 parallel intervention studies (ranging from 5 days to 6 months) and 2 single-arm intervention studies ( 4 and 12 weeks). Only 2 observational studies [26, 27] using estimated self-reported dietary intake data reported candidate BFIs for cocoa and cocoa-product consumption. The most commonly used bio samples were urine $(n=26)$ and plasma $(n=18)$. Urine was collected mainly as 24 -h urine $(n=16)$, but also as spot urine $(n=7)$, as 8 -h urine $(n=1)$ and as 72-h urine $(n=2)$. Number of subjects ranged from 1 up to 59 in the intervention studies and up to 481 for the cross-sectional study. Furthermore, 28 studies used a targeted approach to determine candidate BFIs, and 10 studies used an untargeted approach, by which more compounds could be identified. Among the selected papers, a total of 164 different compounds were found as candidate BFIs for cocoa (products) intake (Additional file 1: Table S1). $( \pm)$-Catechin and (-)-epicatechin derivatives were by far the group of metabolites most reported after cocoa intake, followed by hydroxyphenylvalerolactones, hydroxyphenylvaleric acids and methylxanthines.

\section{Liquorice (products) BFls}

A total of 90 potentially relevant papers were identified from searches in PubMed, Web of Science and Scopus. After a first screening of the title and the abstract, 13 
papers were collected as full text and assessed for further inclusion. Then, 5 papers were excluded due to either inappropriate study designs (e.g. animal studies), unreported liquorice intakes or unreported metabolites/ markers. No additional papers were obtained via hand searches, leading to a total inclusion of 8 papers (Fig. 1). All the included studies were intervention studies to determine candidate BFIs for liquorice (products) (Table 1). In these intervention studies, the participants consumed isolated compounds of liquorice $(n=4)$, solid liquorice $(n=2)$ and liquid liquorice $(n=1)$ or consumed a mixture of a compound or solid liquorice $(n=1)$. In the eight papers, there were four acute single-dose studies, two acute crossover studies, two parallel single-dose studies, two parallel placebo-controlled intervention studies ( 1 and 4 weeks) and one parallel intervention study (5 days). No observational study using estimated self-reported dietary intake data reported candidate BFI liquorice (product) consumption nor were there any longer term studies performed, as the longest study was 4 weeks. Again, plasma and urine were the most commonly used bio samples, $n=7$ and $n=4$, respectively. Multiple spot urine collections were used in two studies, prolonged collection of urine (for 4 and 5 days) was used in two other studies, and one study collected 24-h urine. The number of subjects ranged from 1 up to 60 . Furthermore, all studies used a targeted approach to determine candidate BFIs. Among the selected papers, a total of four different compounds were found as candidate BFIs for liquorice (products) intake (Table 1), namely, 18-glycyrrhetinic acid (18-GA), 18-GA glucuronides, $3 \beta$-monoglucuronyl-18 $\beta$-glycyrrhetinic acid (3-MGA) and glabridin.

\section{Specificity and validity of the identified BFIs Cocoa (products) BFIs}

The specificity of each of the identified candidate BFI was evaluated via a second search step. First, the 164 candidate BFIs were screened for specificity for cocoa or cocoa products in the compound databases HMDB, FooDB and Phenol-Explorer. Based on the presence in other foods, 63 markers were removed from the selection. Three turned out to have an unclear formulation (e.g. missing information on the place of side chains) and were therefore excluded. Based on the search results in the compound databases, the following metabolites were removed because of their presence in other food: epicatechins, catechins and vanillic acid. Important to note is that most of their biotransformation products (e.g. glucuronides and sulfates), a total of 27 metabolites, were not found in the compound databases. As it is known that these biotransformation products are all related to epicatechins, catechins or vannilic acid, they were also removed from the selection. Furthermore, 11 metabolites of caffeine (theobromine, paraxanthine, theophylline, and their biotransformation products) were removed from the selection, since these metabolites are produced in the gut microbiota upon intake of food products containing caffeine, such as coffee, tea or cola (source: HMDB), and they were therefore suspected not to be specific BFIs for chocolate. The specificity of the remaining 61 candidate BFIs was further examined by performing additional searches in Scopus, ISI Web of Science, PubMed or Google Scholar, following the syntax explained in the "Materials and methods" section. Hydroxyphenylvaleric acids and hydroxyphenylvalerolactones were found to be microbial-derived metabolites from polyphenol intake (flavan-3-ols, flavonols and flavanones) [28-30] and were therefore suspected not to be specific BFIs for chocolate intake but markers for all polyphenol-containing foods, such as almonds or tea [28] (removal of 30 metabolites). $N$-PhenylpropenoylL-amino acids are known to be particularly common not only in cocoa but also in coffee and other plant-based foods such as red clover [31, 32] and were therefore removed from the selection (removal of 13 metabolites). Furthermore, a ketone body (3-hydroxybutyrate) and other endogenous metabolites (tyrosine sulfate, $N$-methylguanine, methylglutarylcarnitine, guanidinoacetate) were removed from the selection [27, 33-35]. And lastly, metabolites related to protein intake (xanthurenic acid, indoxyl-sulfate, 4-cresol sulfate [36, 37]), vegetable intake (phenylacetylglutamine [38, 39]), coffee intake (furoylglycine [40]), tea intake (cyclo(Ser-Tyr), cyclo(ProPro) [41]), beer intake (cyclo(Propylalanyl), cyclo(Pro-Pro) [42]), aspartame intake (cyclo(Aspartyl-Phenylalanyl) [43]), nicotinic acid metabolism (hydroxynicotinic acid [44]) and food packaging (di-iso-nonyl phthalate, di-(2-ethylhexyl)phthalate $[45,46])$ were also removed from the selection. This meant that none of the currently identified BFIs for cocoa (products) made the final selection, and therefore, the validity was not checked for any of the candidate BFIs.

\section{Liquorice (products) BFIs}

The specificity of each of the identified candidate BFI for liquorice (products) was also evaluated via a second search step. First, the four candidate BFIs were screened for specificity for liquorice (products) in the compound databases HMDB, FooDB and Phenol-Explorer. The compound 18-GA glucuronide could not be found in any of the compound databases. However, it is known to be the product of the hepatic metabolism of 18-GA [47], and therefore, it was excluded from further evaluation. The other three compounds were found in the databases HMDB and FooDB and were linked to the presence in herbs/spices (18-GA, glabridin) or were known as a sweetener (3-MGA). 3-MGA is also a metabolite of GA 


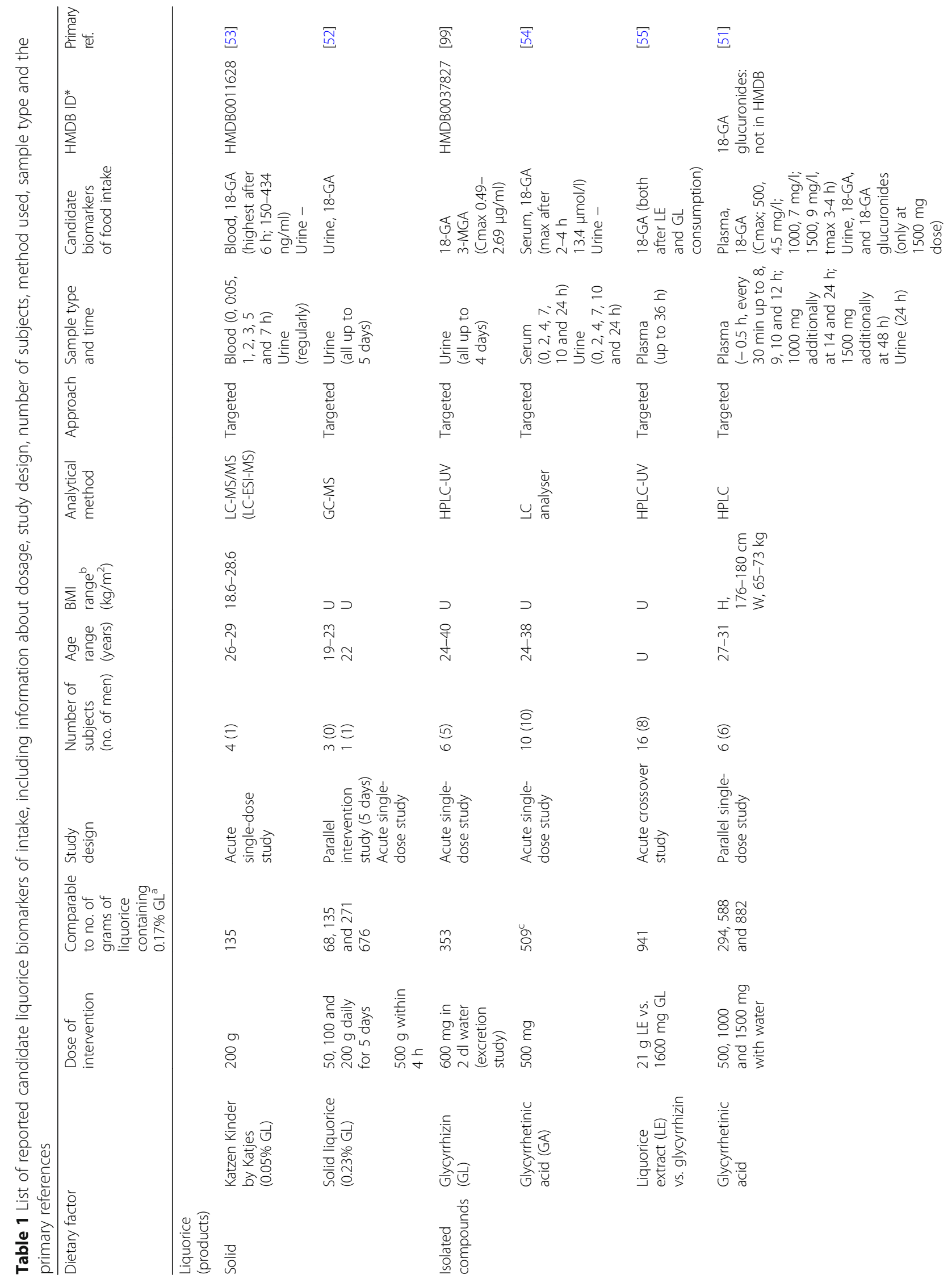




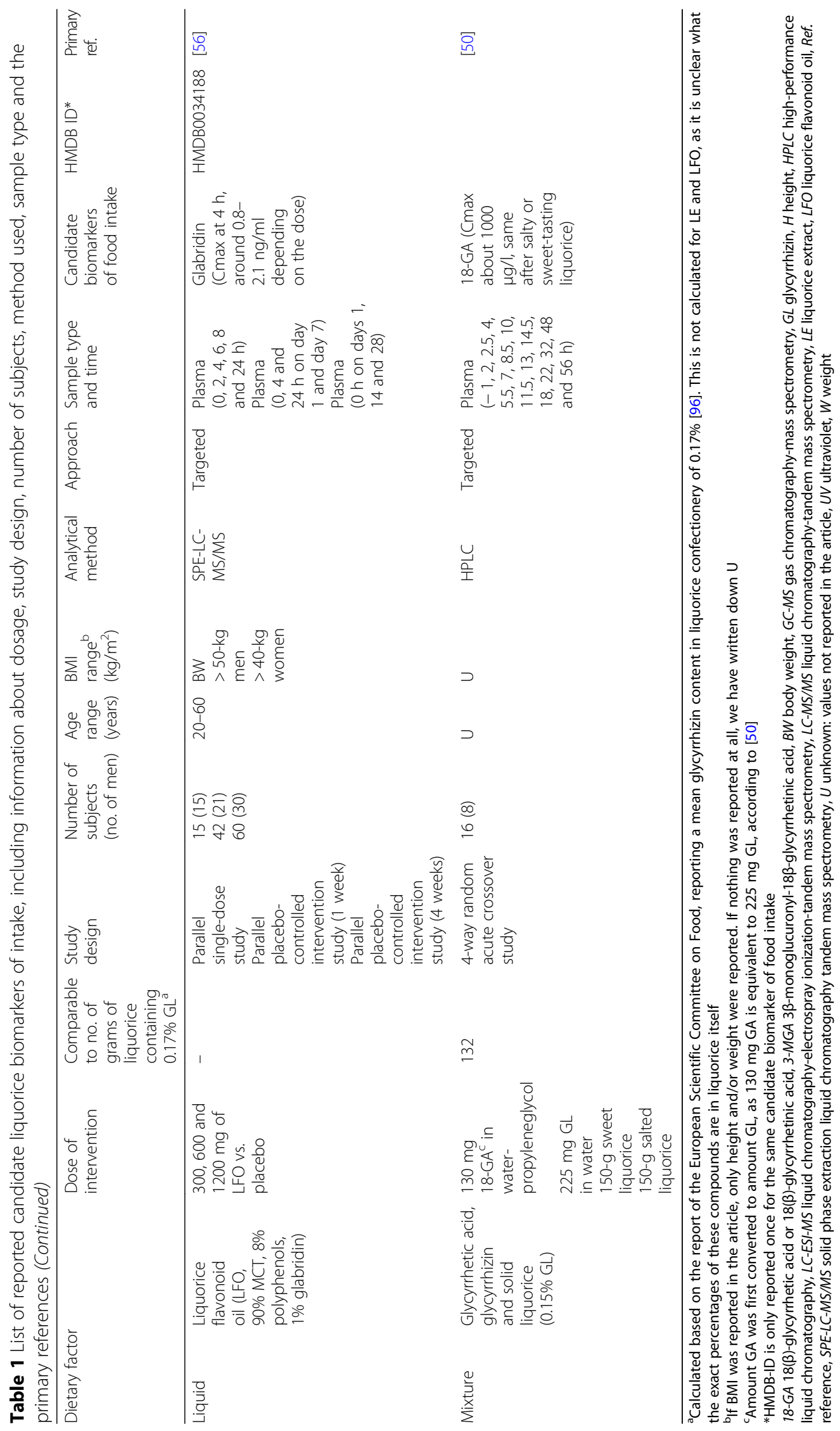


and is known to be excreted via urine in small quantities and to be a possible biomarker for liquorice-induced adverse effects $[19,48]$. Therefore, we decided to include 3-MGA for further evaluation. In Table 1, an overview is presented of the studies on liquorice BFIs, including details on dose used in the study, number of subjects included, which analytical method was used to analyse the samples, which BFIs were found and the primary reference. The specificity of all three candidate BFIs was further examined by performing additional searches in Scopus, ISI Web of Science, PubMed or Google Scholar, which gave us no indication that these metabolites were related to the intake of other food products. On the basis thereof, and on the eight originally included papers, the validity of the three selected candidate BFIs for liquorice (products) was evaluated (Table 2). As can be seen in Table 2, the compound 18-GA in urine after liquorice (product) consumption had the highest assumed validity; however, uncertainty still remains about its specificity, since liquorice root extract itself is used in many other products, such as chewing gum, other confectionary or beverages (question 1) [19, 47, 49, 50]. Krahenbuhl et al. [51] observed a clear dose-response relationship for 18-GA in plasma; however, the concentration GA used in this study was comparable to an intake of liquorice ranging from 1.7 to $5.2 \mathrm{~kg}$; therefore, no relevant intake levels of the targeted food were examined in this study. In the study of Kerstens et al. [52], 50-200 g of liquorice were consumed, showing a dose-response relationship of 18-GA in urine (question 2). 18-GA was found to be traceable in urine after the intake of $50 \mathrm{~g}$ of solid liquorice, and around $0.04 \%$ of the total intake could be traced back after $51 \mathrm{~h}$ in total [52]. A peak in 18-GA concentration was measured after $6 \mathrm{~h}$ in the blood after the consumption of $200 \mathrm{~g}$ of solid liquorice [53], or between 1.5 and $40 \mathrm{~h}$ in urine after the consumption of $600 \mathrm{mg}$ of the compound glycyrrhizin (content comparable to $353 \mathrm{~g}$ of liquorice, Table 1) [19]. 18-GA was also traceable in 24-h urine after the consumption of $1500 \mathrm{mg}$ of the compound GA (content comparable to $882 \mathrm{~g}$ of liquorice, Table 1) [51]. In serum, a peak was observed after 2-4 h of the consumption of $500 \mathrm{mg}$ of the compound GA [54], and in plasma, the peak time was after 3-4 h after the consumption of $21 \mathrm{~g}$ of liquorice extract [55], or $500 \mathrm{mg}$ and higher concentrations of the compound GA [51]. Best sampling time for 3-MGA in urine was unknown, since time to maximum peak height ranged from 1.5 to $39.5 \mathrm{~h}$. This high interindividual variability in time to maximum peak height was likely caused by differences in metabolism rate and enterohepatic cycling of the compound [19] (question 3). None of the studies examined repeated intakes of liquorice, or isolated compounds of liquorice roots (question 4). In plasma, serum and urine, 18-GA was measured after subjects continued with their habitual diet during the measurements (question 5) [50-55]. Since none of the studies was an observational study, or included questionnaire data, the answers to question 6 were "no" for all candidate BFIs. Kerstens et al. [52] did examine whether 18-GA could be used to detect whether two patients had consumed liquorice, even if the patients denied having eaten liquorice-containing products. They observed that it was indeed possible to detect liquorice intake via measuring $18-\mathrm{GA}$ in urine, after which the patient indeed admitted to have eaten liquorice containing products. Unfortunately, data on actual liquorice intake (via for example food frequency questionnaires or 24-h recalls) were not recorded in this study. Question 7 concerned the chemical and biological stability during specimen collection and storage. In the

Table 2 Evaluation of the validity of the identified candidate biomarkers of food intake for liquorice

\begin{tabular}{|c|c|c|c|c|c|c|c|c|c|c|c|c|}
\hline Metabolite & Bio fluid & Q1 & Q2 & Q3 & Q4 & Q5 & Q6 & Q7 & Q8 & Q9 & Sum & References \\
\hline \multicolumn{13}{|l|}{ Candidate liquorice biomarkers of food intake } \\
\hline \multirow[t]{4}{*}{ 18-glycyrrhetic acid } & Plasma & $U$ & $U$ & Y & $U$ & Y & N & $U$ & Y & N & 3 & {$[50,51,55]$} \\
\hline & Serum & $U$ & $U$ & Y & $U$ & Y & N & $U$ & Y & N & 3 & [54] \\
\hline & Blood & $U$ & $U$ & Y & $U$ & $\mathrm{~N}$ & N & $U$ & Y & N & 2 & [53] \\
\hline & Urine & $U$ & Y & Y & $U$ & Y & N & $U$ & Y & N & 4 & [51-53] \\
\hline $3 \beta$-monoglucuronyl-18ß-glycyrrhetinic acid & Urine & U & U & N & $U$ & Y & N & $U$ & Y & N & 2 & [19] \\
\hline Glabridin & Plasma & $U$ & $U$ & $U$ & $U$ & $\mathrm{~N}$ & $\mathrm{~N}$ & $U$ & $\mathrm{~N}$ & $\mathrm{~N}$ & 0 & [56] \\
\hline
\end{tabular}

$Y$ yes, $N$ no, $U$ unknown

Q1: Is the marker compound plausible as a specific BFI for the food or food group (chemical/biological plausibility)?

Q2: Is there a dose-response relationship at relevant intake levels of the targeted food (quantitative aspect)?

Q3: Is the single-meal time-response relationship described adequately to make a wise choice of sample type and time window (single-dose kinetics)?

Q4: Is the biomarker kinetics for repeated intakes of the food/food group described adequately providing the frequency of sampling needed to assess habitual intake (e.g. cumulative aspects)?

Q5: Has the marker been shown to be robust after intake of complex meals reflecting dietary habits of the targeted population (robustness)? Q6: Has the marker been shown to compare well with other markers or questionnaire data for the same food/food group (reliability)?

Q7: Is the marker chemically and biologically stable during bio specimen collection and storage, making measurements reliable and feasible?

Q8: Are analytical variability (CV\%), accuracy, sensitivity and specificity known as adequate for at least one reported analytical method?

Q9: Has the analysis been successfully reproduced in another laboratory (reproducibility)? 
eight included papers, it was described that urine samples were stored at $4{ }^{\circ} \mathrm{C}[19,54]$ or $-20{ }^{\circ} \mathrm{C}[52,53]$, and blood, plasma and serum samples were stored at $-20{ }^{\circ} \mathrm{C}$ $[50-53,55]$ before continuing with the analyses. In some cases, no information was available about the storage temperature of the samples [56], and for all eight studies, it was unclear how long the samples had been stored. Analytical variability for measuring 18-GA in plasma was 3\% [51]; in urine, CV\% was between 6 and $9.3 \%[51,53]$; in serum, there was a $2.1 \%$ within assay variability and $8.5 \%$ between assay variability [54]; and in blood, CV\% was between 4.6 and 6.3\% [53]. For 3-MGA, the repeatability of the measurements within and between days was tested, with peak height 4.88 and 7.21 RSD\%, respectively, and peak area 2.61 and 6.21 RSD\%, respectively [19]. In the papers of Raggi et al. [55], Aoki et al. [56] and Ploeger et al. [50], no details about analytical variability were presented (question 8). Lastly, none of the analyses described in the eight selected papers were reproduced by another laboratory. The analyses used were either described in the paper for the first time $[19,50,52,53,55]$ or had previously been performed at their own lab $[51,54,56]$ (question 9).

\section{Discussion}

The present systematic review examined the current status of candidate BFIs for the consumption of cocoa (products) and liquorice. In total, 37 relevant papers were included for cocoa (products) [26, 27, 32-34, 44, 57-87], and 8 relevant papers were included for liquorice (products) [19, 50-56]. For cocoa (products), 164 different compounds were identified as candidate BFIs in the 37 obtained papers. After evaluating the specificity of these compounds, none of these candidate BFIs turned out to be specific for cocoa (products). Therefore, the validity of these compounds was not further examined. For liquorice (products), 4 different compounds were identified as candidate BFIs in the 8 obtained papers. After evaluating the specificity and the validity of these 4 compounds, 18-GA in urine was found to have the highest assumed validity.

Regarding the 164 cocoa (products) BFIs (Additional file 1: Table S1), none of the identified compounds was specific. Our results indicated that most of the identified BFIs for cocoa (products) were also found or are expected to be found after the consumption of foods such as tea, coffee or red wine. This is due to similarities in the composition of these foods, such as a high polyphenol content or the presence of caffeine compounds [88-91]. Only a few randomised trials (both short and long term) have compared the metabolite profile after consumption of cocoa (products) with other products showing a similar metabolite profile, such as the metabolite profiles observed after tea $[63,71]$ or coffee [63]. Up till now, no metabolites were obtained that could discriminate between these food products. However, only a small amount of metabolites was measured in these studies via targeted approaches. With untargeted approaches, a higher number of compounds can be measured in bio fluids and therefore might elucidate compounds that are specific and valid BFIs for cocoa (products). Before any conclusion can be drawn about the specificity of the identified BFIS for cocoa (products), more randomized intervention studies comparing food products with a similar metabolite excretion pattern, using untargeted approaches, are needed. Furthermore, it remains to be explored whether the dose-response relationship is equal after consuming for example same amounts of tea and a cocoa drink [63]. Another important point to consider is that the way of processing of the cocoa beans, the cocoa variety and the origin of the cocoa bean can affect the final concentration of compounds in the cocoa product [92]. In addition, cocoa beans are subject to seasonal variation, again affecting concentrations of several compounds in the beans [93, 94]. In this review, we focused on single BFIs for cocoa intake, which turned out to be nonspecific. A combination of several biomarkers, a so-called biomarker profile or panel of biomarkers might however increase the specificity for cocoa intake. Garcia-Aloy and colleagues [95] have examined this possibility in an untargeted study and found a combined model for cocoa consumption that included 7-methylxanthine and dihydroxyphenylvalerolactone glucuronide. This combined model was a better discriminant for cocoa consumption compared to all individual metabolites. It is essential to explore whether this combination of compounds will also discriminate cocoa (products) intake from tea intake or coffee intake.

The four identified liquorice BFIs (Table 1), 18-GA, 18-GA glucuronides, 3-MGA and glabridin, were only described in relation to liquorice intake or liquorice root extracts. However, liquorice root extracts are known to be used in a variety of products, such as different sweets, chewing gums, chewing tobacco and tea, or even in (alcoholic) drinks and medicinal products, often as a sweetening or flavouring agent $[19,47,49,50]$. It will therefore be necessary to examine the relative content and contribution of liquorice extract in these products and in the habitual consumed diets overall. An important point to consider is that the content of liquorice root extracts may vary from product to product, depending on the characteristics the producer desires for that product, which causes variation in the final concentrations of liquorice root extracts in these products. For example, in the solid liquorice that was consumed in the included studies, the GL content varied from 0.05 up to $0.23 \%$. 18-GA in urine was the most promising and most 
studied candidate BFI out of the three candidates for liquorice (products). To increase its validity, studies on repeated intake, habitual food consumption, dietary patterns, stability during storage and reproducibility of the methods between labs are still needed. Important to note is that in all studies examining liquorice biomarkers, subjects consumed $68 \mathrm{~g}$ of liquorice or more (when standardized to the average glycyrrhizin content in liquorice confectionary of $0.17 \%$ [96], this is equivalent to an intake of GL of $116 \mathrm{mg}$ ), while the European Scientific Committee on Food advises that ingestion of liquorice should not exceed $58 \mathrm{~g}$ per day (equivalent to an intake of $100 \mathrm{mg}$ GL per day) [96]. Therefore, it is crucial to investigate whether 18-GA is still a reliable biomarker when measured after the intake of a low dose of liquorice, or after repeated intake of low doses of liquorice. This is especially important since some studies were unable to measure 18-GA in urine, because the amount was below the detection limit at lower concentrations of intake $[51,54]$. Clearly lacking in the literature for all candidate BFIs for liquorice were long-term intervention studies, dietary pattern studies and observational studies. Currently, the longest study had a duration of 4 weeks. However, this study was done using liquorice flavonoid oil in which glycyrrhizin was almost removed from the product $(<0.005 \%)$ [56]. Consequently, this study did not give us information about the consumption of solid liquorice or other products using liquorice root extracts, which have a higher glycyrrhizin content. The lack of observational and dietary pattern studies makes it impossible to properly validate any of the three found candidate BFIs; therefore, these studies are urgently needed. Moreover, we only found the polyphenol glabridin as candidate BFI for liquorice intake; however, there are more polyphenols known to be present in liquorice roots. For example, Vaya et al. [97] have isolated hispaglabridin A, hispaglabridin B, 4'-O-methylglabridin, formononetin and glabridin itself from liquorice roots. It should be further examined what the exact contribution of these polyphenols is in liquorice (products) and whether these compounds could be possible BFIs for liquorice (products). For 18-GA and glabridin, commercial standards are available; for 18-GA also, a non-commercial standard is available through FoodComEx (Food Compound Exchange, foodcomex.org), an online catalogue of pure compounds made available by academic laboratories [98].

\section{Conclusions}

In this paper, we have identified potential BFIs for cocoa (products) and liquorice (products). For cocoa (products), none of the individual BFIs were found to be specific. However, a combination of individual BFIs might lead to discriminating profiles between cocoa (products) and foods with a similar composition. This needs to be further explored. We did identify 18-GA as a promising candidate BFI for liquorice; however, important information on its validity is still missing, and therefore, more research is needed. This systematic review shows that there is still an urgent need for research to identify specific and valid biomarkers of the consumption of cocoa (products) and liquorice (products).

\section{Additional file}

Additional file 1: Table S1. Overview of the studies on biomarkers for cocoa and chocolate, including information about dosage, study design, number of subjects, method used, sample type and the original references. (DOCX $102 \mathrm{~kb}$ )

\section{Abbreviations}

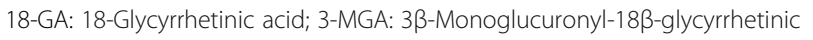
acid; BFIRev: Biomarker of Food Intake Reviews; BFIs: Biomarkers of food intake; FooDB: Food DataBase; FoodBAll: Food Biomarkers Alliance; FoodComEx: Food Compound Exchange; HMDB: Human Metabolome DataBase; HOMA-IR: Homeostatic Model Assessment-Insulin Resistance

\section{Acknowledgements}

All FoodBAll collaborators and the EU Joint Programming Initiative "A Healthy Diet for a Healthy Life" are gratefully acknowledged.

\section{Funding}

The project was funded by the BioNH call (grant number 529051002) under the Joint Programming Initiative, "A Healthy Diet for a Healthy Life" (JPI HDHL, website: http://www.healthydietforhealthylife.eu) [grant number FOODBALLPCIN-2014-133]; the Spanish Ministry of the Economy and Competitiveness (MINECO) (PCIN-2014-133-MINECO, Spain) together with the Joint Programming Initiative "A Healthy Diet for a Healthy Life"; CIBERFES (co-funded by the FEDER Program from the EU); and the award of the Generalitat de Catalunya's Agency AGAUR [grant number 2014SGR1566]. EAA would like to thank CONACYT (Mexico) for the PhD fellowship. MU-S would like to thank the "Ramón y Cajal" program from MINECO and the Fondo Social Europeo.

\section{Authors' contributions}

CCJRM performed systematic database search; selected papers and critically reviewed whether they adhered to the selection criteria, on both the cocoa and liquorice part; and wrote the manuscript. EAA selected papers and critically reviewed whether they adhered to the selection criteria, of the cocoa part, and critically revised the manuscript. EMBB selected papers and critically reviewed whether they adhered to the selection criteria of the liquorice part. MUS and LAA critically revised the manuscript for important intellectual content. All authors read and approved the final manuscript.

\section{Ethics approval and consent to participate}

Not applicable

\section{Consent for publication}

Not applicable

\section{Competing interests}

The authors declare that they have no competing interests.

\section{Publisher's Note}

Springer Nature remains neutral with regard to jurisdictional claims in published maps and institutional affiliations.

\section{Author details}

${ }^{1}$ Division of Human Nutrition and Health, Wageningen University and Research Centre, Stippeneng 4, 6708 WE Wageningen, The Netherlands. ${ }^{2}$ Department of Nutrition, Food Sciences and Gastronomy, Biomarkers and 
Nutrimetabolomics Laboratory, XaRTA, INSA-UB, Faculty of Pharmacy and Food Sciences, University of Barcelona, Barcelona, Spain. ${ }^{3}$ CIBER Fragilidad y Envejecimiento Saludable (CIBERFES), Instituto de Salud Carlos III, 08028 Barcelona, Spain

\section{Received: 28 March 2018 Accepted: 5 July 2018}

Published online: 27 July 2018

\section{References}

1. Buijsse B, Feskens EM, Kok FJ, Kromhout D. Cocoa intake, blood pressure, and cardiovascular mortality: the Zutphen Elderly Study. Arch Intern Med. 2006;166:411-7.

2. Alkerwi AA, Sauvageot N, Crichton GE, Elias MF, Stranges S. Daily chocolate consumption is inversely associated with insulin resistance and liver enzymes in the Observation of Cardiovascular Risk Factors in Luxembourg study. Br J Nutr. 2016;115:1661-8.

3. Djousse L, Hopkins PN, Arnett DK, Pankow JS, Borecki I, North KE, et al. Chocolate consumption is inversely associated with calcified atherosclerotic plaque in the coronary arteries: the NHLBI Family Heart Study. Clin Nutr. 2011;30:38-43.

4. Buijsse B, Weikert C, Drogan D, Bergmann M, Boeing H. Chocolate consumption in relation to blood pressure and risk of cardiovascular disease in German adults. Eur Heart J. 2010;31:1616-23.

5. Janszky I, Mukamal K, Ljung R, Ahnve S, Ahlbom A, Hallqvist J. Chocolate consumption and mortality following a first acute myocardial infarction: the Stockholm Heart Epidemiology Program. J Intern Med. 2009;266:248-57.

6. Hooper L, Kay C, Abdelhamid A, Kroon PA, Cohn JS, Rimm EB, et al. Effects of chocolate, cocoa, and flavan-3-ols on cardiovascular health: a systematic review and meta-analysis of randomized trials. Am J Clin Nutr. 2012:95:740-51.

7. Fiore C, Eisenhut M, Ragazzi E, Zanchin G, Armanini D. A history of the therapeutic use of liquorice in Europe. J Ethnopharmacol. 2005;99:317-24.

8. Xiaoying W, Han Z, Yu W: Glycyrrhiza glabra (Licorice): ethnobotany and health benefits. In Sustained energy for enhanced human functions and activity. United States: Elsevier; 2018: 231-50.

9. Johnson L, Mander AP, Jones LR, Emmett PM, Jebb SA. Energy-dense, low-fiber, high-fat dietary pattern is associated with increased fatness in childhood. Am J Clin Nutr. 2008;87:846-54.

10. Nishida C, Uauy R, Kumanyika S, Shetty P. The joint WHO/FAO expert consultation on diet, nutrition and the prevention of chronic diseases: process, product and policy implications. Public Health Nutr. 2004;7:245-50.

11. Van Uum SH. Liquorice and hypertension. Neth J Med. 2005;63:119-20.

12. Shintani S, Murase H, Tsukagoshi H, Shiigai T. Glycyrrhizin (licorice)-induced hypokalemic myopathy. Eur Neurol. 1992;32:44-51.

13. Favé G, Beckmann M, Lloyd AJ, Zhou S, Harold G, Lin W, et al. Development and validation of a standardized protocol to monitor human dietary exposure by metabolite fingerprinting of urine samples. Metabolomics. 2011:7:469-84

14. Jenab M, Slimani N, Bictash M, Ferrari P, Bingham SA. Biomarkers in nutritional epidemiology: applications, needs and new horizons. Hum Genet. 2009;125:507-25.

15. Brennan L, Gibbons H, O'Gorman A. An overview of the role of metabolomics in the identification of dietary biomarkers. Curr Nutr Reports. 2015:4:304-12

16. O'Gorman A, Gibbons H, Brennan L. Metabolomics in the identification of biomarkers of dietary intake. Comput Struct Biotechnol J. 2013;4: e201301004.

17. Dragsted LO, Gao Q, Praticò G, Manach C, Wishart DS, Scalbert A, et al. Dietary and health biomarkers - time for an update. Genes Nutr. 2017;12:24.

18. Gao Q, Praticò G, Scalbert A, Vergères $G$, Kolehmainen M, Manach C, et al. A scheme for a flexible classification of dietary and health biomarkers. Genes Nutr. 2017;12:34.

19. Glavac NK, Kreft S. Excretion profile of glycyrrhizin metabolite in human urine. Food Chem. 2012;131:305-8.

20. Lloyd AJ, Favé G, Beckmann M, Lin W, Tailliart K, Xie L, et al. Use of mass spectrometry fingerprinting to identify urinary metabolites after consumption of specific foods. Am J Clin Nutr. 2011:94:981-91.

21. Scalbert A, Brennan L, Manach C, Andres-Lacueva C, Dragsted LO, Draper J, et al. The food metabolome: a window over dietary exposure. Am J Clin Nutr. 2014;99:1286-308.
22. Praticò G, Gao Q, Scalbert A, Vergères G, Kolehmainen M, Manach C, et al. Guidelines for Biomarker of Food Intake Reviews (BFIRev): how to conduct an extensive literature search for biomarker of food intake discovery. Genes Nutr. 2018;13:3.

23. Moher D, Liberati A, Tetzlaff J, Altman DG. Preferred reporting items for systematic reviews and meta-analyses: the PRISMA statement. Ann Intern Med. 2009;151:264-9.

24. Ottaviani Jl, Borges G, Momma TY, Spencer JPE, Keen CL, Crozier A, et al. The metabolome of 2-C-14 (-)-epicatechin in humans: implications for the assessment of efficacy, safety, and mechanisms of action of polyphenolic bioactives. Sci Rep. 2016;6:29304/1-29304/10

25. Dragsted LO, Gao Q, Scalbert A, Vergères G, Kolehmainen M, Manach C, et al. Validation of biomarkers of food intake-critical assessment of candidate biomarkers. Genes Nutr. 2018;13:14.

26. Edmands WM, Ferrari P, Rothwell JA, Rinaldi S, Slimani N, Barupal DK, et al. Polyphenol metabolome in human urine and its association with intake of polyphenol-rich foods across European countries. Am J Clin Nutr. 2015;102:905-13.

27. Garcia-Aloy M, Llorach R, Urpi-Sarda M, Jauregui O, Corella D, Ruiz-Canela M, et al. A metabolomics-driven approach to predict cocoa product consumption by designing a multimetabolite biomarker model in free-living subjects from the PREDIMED study. Mol Nutr Food Res. 2015;59:212-20.

28. Garrido I, Urpi-Sarda M, Monagas M, Gómez-Cordovés C, Martín-Álvarez PJ, Llorach $\mathrm{R}$, et al. Targeted analysis of conjugated and microbial-derived phenolic metabolites in human urine after consumption of an almond skin phenolic extract-3. J Nutr. 2010;140:1799-807.

29. Urpi-Sarda M, Garrido I, Monagas M, Gómez-Cordovés C, Medina-Remón A, Andres-Lacueva C, et al. Profile of plasma and urine metabolites after the intake of almond [Prunus dulcis (Mill.) D.A. Webb] polyphenols in humans. J Agric Food Chem. 2009;57:10134-42.

30. Fraga CG. Plant phenolics and human health. Hoboken: John Wiley and Sons, Inc:; 2010

31. El-Seedi HR, El-Said AMA, Khalifa SAM, Göransson U, Bohlin L, Borg-Karlson A-K, et al. Biosynthesis, natural sources, dietary intake, pharmacokinetic properties, and biological activities of hydroxycinnamic acids. J Agric Food Chem. 2012;60:10877-95

32. Stark T, Lang R, Keller D, Hensel A, Hofmann T. Absorption of Nphenylpropenoyl-L-amino acids in healthy humans by oral administration of cocoa (Theobroma cacao). Mol Nutr Food Res. 2008:52:1201-14.

33. Llorach R, Urpi-Sarda M, Tulipani S, Garcia-Aloy M, Monagas M, AndresLacueva C. Metabolomic fingerprint in patients at high risk of cardiovascular disease by cocoa intervention. Mol Nutr Food Res. 2013;57:962-73.

34. Llorach-Asuncion R, Jauregui O, Urpi-Sarda M, Andres-Lacueva C. Methodological aspects for metabolome visualization and characterization: a metabolomic evaluation of the $24 \mathrm{~h}$ evolution of human urine after cocoa powder consumption. J Pharm Biomed Anal. 2010;51:373-81.

35. Peters BA, Hall MN, Liu X, Parvez F, Siddique AB, Shahriar H, et al. Low-dose creatine supplementation lowers plasma guanidinoacetate, but not plasma homocysteine, in a double-blind, randomized, placebo-controlled trial-3. J Nutr. 2015;145:2245-52.

36. Hjerpsted JB, Ritz C, Schou SS, Tholstrup T, Dragsted LO. Effect of cheese and butter intake on metabolites in urine using an untargeted metabolomics approach. Metabolomics. 2014;10:1176-85.

37. Patel KP, Luo FJG, Plummer NS, Hostetter TH, Meyer TW. The production of $\mathrm{p}$-cresol sulfate and indoxyl sulfate in vegetarians versus omnivores. Clin J Am Soc Nephrol. 2012;7:982-8.

38. O'Sullivan A, Gibney MJ, Brennan L. Dietary intake patterns are reflected in metabolomic profiles: potential role in dietary assessment studies. Am J Clin Nutr. 2010;93:314-21.

39. Heinzmann SS, Merrifield CA, Rezzi S, Kochhar S, Lindon JC, Holmes E, et al. Stability and robustness of human metabolic phenotypes in response to sequential food challenges. J Proteome Res. 2011;11:643-55.

40. Heinzmann SS, Holmes E, Kochhar S, Nicholson JK, Schmitt-Kopplin P. 2-Furoylglycine as a candidate biomarker of coffee consumption. J Agric Food Chem. 2015:63:8615-21.

41. Yamamoto K, Hayashi M, Murakami Y, Araki Y, Otsuka Y, Kashiwagi T, et al. Development of LC-MS/MS analysis of cyclic dipeptides and its application to tea extract. Biosci Biotechnol Biochem. 2016;80:172-7.

42. Gautschi M, Schmid JP, Peppard TL, Ryan TP, Tuorto RM, Yang X. Chemical characterization of diketopiperazines in beer. J Agric Food Chem. 1997;45:3183-9.

43. Lipton WE, Li Y-N, Younoszai MK, Stegink LD. Intestinal absorption of aspartame decomposition products in adult rats. Metabolism. 1991;40:1337-45. 
44. Llorach R, Urpi-Sarda M, Jauregui O, Monagas M, Andres-Lacueva C. An LCMS-based metabolomics approach for exploring urinary metabolome modifications after cocoa consumption. J Proteome Res. 2009;8:5060-8.

45. Larsson K, Bjorklund KL, Palm B, Wennberg M, Kaj L, Lindh CH, et al. Exposure determinants of phthalates, parabens, bisphenol $\mathrm{A}$ and triclosan in Swedish mothers and their children. Environ Int. 2014;73:323-33.

46. Ackerman JM, Dodson RE, Engel CL, Gray JM, Rudel RA. Temporal variability of urinary di (2-ethylhexyl) phthalate metabolites during a dietary intervention study. J Expo Sci Environ Epidemiol. 2014;24:595.

47. Ploeger B, Mensinga T, Sips A, Seinen W, Meulenbelt J, DeJongh J. The pharmacokinetics of glycyrrhizic acid evaluated by physiologically based pharmacokinetic modeling. Drug Metab Rev. 2001;33:125-47.

48. Makino T. 3-Monoglucuronyl glycyrrhretinic acid is a possible marker compound related to licorice-induced pseudoaldosteronism. Biol Pharm Bull. 2014;37:898-902.

49. Omar HR, Komarova I, El-Ghonemi M, Fathy A, Rashad R, Abdelmalak HD, et al. Licorice abuse: time to send a warning message. Ther Adv Endocrinol Metab. 2012;3:125-38.

50. Ploeger B, Mensinga T, Sips A, Meulenbelt J, DeJongh J. A human physiologicallybased model for glycyrrhzic acid, a compound subject to presystemic metabolism and enterohepatic cycling. Pharm Res. 2000;17:1516-25.

51. Krahenbuhl S, Hasler F, Frey BM, Frey FJ, Brenneisen R, Krapf R. Kinetics and dynamics of orally administered 18 beta-glycyrrhetinic acid in humans. J Clin Endocrinol Metab. 1994;78:581-5.

52. Kerstens MN, Guillaume CPF, Wolthers BG, Dullaart RPF. Gas chromatographic-mass spectrometric analysis of urinary glycyrrhetinic acid: an aid in diagnosing liquorice abuse. J Intern Med. 1999;246:539-47.

53. Albermann ME, Musshoff F, Hagemeier L, Madea B. Determination of glycyrrhetic acid after consumption of liquorice and application to a fatality. Forensic Sci Int. 2010;197:35-9.

54. Heilman P, Heide J, Hundertmark S, Schöneshöfer M. Administration of glycyrrhetinic acid: significant correlation between serum levels and the cortisol/cortisone-ratio in serum and urine. Exp Clin Endocrinol Diabetes. 1999:107:370-8

55. Raggi MA, Maffei F, Bugamelli F, Cantelli Forti G. Bioavailability of glycyrrhizin and licorice extract in rat and human plasma as detected by a HPLC method. Pharmazie. 1994;49:269-72.

56. Aoki F, Nakagawa K, Kitano M, Ikematsu H, Nakamura K, Yokota S, et al. Clinical safety of licorice flavonoid oil (Ifo) and pharmacokinetics of glabridin in healthy humans. J Am Coll Nutr. 2007;26:209-18.

57. Actis-Goretta L, Leveques A, Giuffrida F, Destaillats F, Nagy K. Identification of O-methyl-(-)-epicatechin-O-sulphate metabolites by mass-spectrometry after O-methylation with trimethylsilyldiazomethane. J Chromatogr A. 2012; 1245:150-7.

58. Actis-Goretta $L$, Lévèques $A$, Giuffrida $F$, Romanov-Michailidis $F$, Viton $F$, Barron D, et al. Elucidation of (-)-epicatechin metabolites after ingestion of chocolate by healthy humans. Free Radic Biol Med. 2012;53:787-95.

59. Andersen MBS, Kristensen M, Manach C, Pujos-Guillot E, Poulsen SK, Larsen $\mathrm{TM}$, et al. Discovery and validation of urinary exposure markers for different plant foods by untargeted metabolomics. Anal Bioanal Chem. 2014;406: 1829-44.

60. Andersen MB, Rinnan A, Manach C, Poulsen SK, Pujos-Guillot E, Larsen TM, et al. Untargeted metabolomics as a screening tool for estimating compliance to a dietary pattern. J Proteome Res. 2014;13:1405-18.

61. Baba S, Osakabe N, Yasuda A, Natsume M, Takizawa T, Nakamura T, et al. Bioavailability of (-)-epicatechin upon intake of chocolate and cocoa in human volunteers. Free Radic Res. 2000;33:635-41.

62. Holt RR, Lazarus SA, Sullards MC, Zhu QY, Schramm DD, Hammerstone JF, et al. Procyanidin dimer B2 [epicatechin-(4beta-8)-epicatechin] in human plasma after the consumption of a flavanol-rich cocoa. Am J Clin Nutr. 2002; 76:798-804

63. Ito H, Gonthier MP, Manach C, Morand C, Mennen L, Remesy C, et al. Polyphenol levels in human urine after intake of six different polyphenolrich beverages. Br J Nutr. 2005;94:500-9.

64. Martin FP, Montoliu I, Nagy K, Moco S, Collino S, Guy P, et al. Specific dietary preferences are linked to differing gut microbial metabolic activity in response to dark chocolate intake. J Proteome Res. 2012;11:6252-63.

65. Martínez-López S, Sarriá B, Gómez-Juaristi M, Goya L, Mateos R, BravoClemente L. Theobromine, caffeine, and theophylline metabolites in human plasma and urine after consumption of soluble cocoa products with different methylxanthine contents. Food Res Int. 2014;63:446-55.
66. Mullen W, Borges G, Donovan JL, Edwards CA, Serafini M, Lean ME, et al. Milk decreases urinary excretion but not plasma pharmacokinetics of cocoa flavan-3-ol metabolites in humans. Am J Clin Nutr. 2009;89:1784-91.

67. Neilson AP, George JC, Janle EM, Mattes RD, Rudolph R, Matusheski NV, et al. Influence of chocolate matrix composition on cocoa flavan-3-ol bioaccessibility in vitro and bioavailability in humans. J Agric Food Chem. 2009;57:9418-26.

68. Ottaviani Jl, Balz M, Kimball J, Ensunsa JL, Fong R, Momma TY, et al. Safety and efficacy of cocoa flavanol intake in healthy adults: a randomized, controlled, double-masked trial. Am J Clin Nutr. 2015;102:1425-35

69. Ottaviani JI, Momma TY, Kuhnle GK, Keen CL, Schroeter H. Structurally related (-)-epicatechin metabolites in humans: assessment using de novo chemically synthesized authentic standards. Free Radic Biol Med. 2012;52: 1403-12.

70. Rein D, Lotito S, Holt RR, Keen CL, Schmitz HH, Fraga CG. Epicatechin in human plasma: in vivo determination and effect of chocolate consumption on plasma oxidation status. J Nutr. 2000;130:2109s-14s.

71. Richelle M, Tavazzi I, Enslen M, Offord EA. Plasma kinetics in man of epicatechin from black chocolate. Eur J Clin Nutr. 1999;53:22-6.

72. Rios LY, Bennett RN, Lazarus SA, Rémésy C, Scalbert A, Williamson G. Cocoa procyanidins are stable during gastric transit in humans. Am J Clin Nutr. 2002;76:1106-10.

73. Rios LY, Gonthier MP, Remesy C, Mila I, Lapierre C, Lazarus SA, et al. Chocolate intake increases urinary excretion of polyphenol-derived phenolic acids in healthy human subjects. Am J Clin Nutr. 2003;77:912-8.

74. Ritter C, Zimmermann BF, Galensa R. Chiral separation of (+)/(-)-catechin from sulfated and glucuronidated metabolites in human plasma after cocoa consumption. Anal Bioanal Chem. 2010;397:723-30.

75. Rodriguez-Mateos A, Cifuentes-Gomez T, Gonzalez-Salvador I, Ottaviani Jl, Schroeter $\mathrm{H}$, Kelm M, et al. Influence of age on the absorption, metabolism, and excretion of cocoa flavanols in healthy subjects. Mol Nutr Food Res. 2015:59:1504-12.

76. Rodriguez-Mateos A, Oruna-Concha MJ, Kwik-Uribe C, Vidal A, Spencer JP. Influence of sugar type on the bioavailability of cocoa flavanols. Br J Nutr. 2012;108:2243-50.

77. Roura E, Almajano MP, Bilbao MLM, Andrés-Lacueva C, Estruch R, LamuelaRaventós RM. Human urine: epicatechin metabolites and antioxidant activity after cocoa beverage intake. Free Radic Res. 2007:41:943-9.

78. Roura E, Andres-Lacueva C, Estruch R, Lourdes Mata Bilbao M, IzquierdoPulido M, Lamuela-Raventos RM. The effects of milk as a food matrix for polyphenols on the excretion profile of cocoa (-)-epicatechin metabolites in healthy human subjects. Br J Nutr. 2008;100:846-51.

79. Roura $E$, Andrés-Lacueva $C$, Jáuregui $O$, Badia E, Estruch $R$, Izquierdo-Pulido $\mathrm{M}$, et al. Rapid liquid chromatography tandem mass spectrometry assay to quantify plasma (-)-epicatechin metabolites after ingestion of a standard portion of cocoa beverage in humans. J Agric Food Chem. 2005;53:6190-4.

80. Roura E, Andres-Lacueva C, Estruch R, Mata-Bilbao ML, Izquierdo-Pulido M, Waterhouse AL, et al. Milk does not affect the bioavailability of cocoa powder flavonoid in healthy human. Ann Nutr Metab. 2007;51:493-8.

81. Schroeter $\mathrm{H}$, Heiss C, Balzer J, Kleinbongard P, Keen CL, Hollenberg NK, et al. (-)-Epicatechin mediates beneficial effects of flavanol-rich cocoa on vascular function in humans. Proc Natl Acad Sci U S A. 2006;103:1024-9.

82. Shively CA, Tarka SM, Arnaud MJ, Dvorchik BH, Passananti GT, Vesell ES. High levels of methylxanthines in chocolate do not alter theobromine disposition. Clin Pharmacol Ther. 1985;37:415-24.

83. Urpi-Sarda M, Llorach R, Khan N, Monagas M, Rotches-Ribalta M, LamuelaRaventos $\mathrm{R}$, et al. Effect of milk on the urinary excretion of microbial phenolic acids after cocoa powder consumption in humans. J Agric Food Chem. 2010;58:4706-11.

84. Urpi-Sarda M, Monagas M, Khan N, Lamuela-Raventos RM, Santos-Buelga C, Sacanella E, et al. Epicatechin, procyanidins, and phenolic microbial metabolites after cocoa intake in humans and rats. Anal Bioanal Chem. 2009;394:1545-56.

85. Urpi-Sarda M, Monagas M, Khan N, Llorach R, Lamuela-Raventós RM, Jáuregui $\mathrm{O}$, et al. Targeted metabolic profiling of phenolics in urine and plasma after regular consumption of cocoa by liquid chromatographytandem mass spectrometry. J Chromatogr A. 2009;1216:7258-67.

86. Vitaglione P, Barone Lumaga R, Ferracane R, Sellitto S, Morello JR, Reguant Miranda J, et al. Human bioavailability of flavanols and phenolic acids from cocoa-nut creams enriched with free or microencapsulated cocoa polyphenols. Br J Nutr. 2013;109:1832-43. 
87. Ibero-Baraibar I, Romo-Hualde A, Gonzalez-Navarro CJ, Zulet MA, Martinez JA. The urinary metabolomic profile following the intake of meals supplemented with a cocoa extract in middle-aged obese subjects. Food Funct. 2016;7:1924-31.

88. Appeldoorn MM, Vincken J-P, Aura A-M, Hollman PCH, Gruppen H. Procyanidin dimers are metabolized by human microbiota with 2-(3,4-

dihydroxyphenyl)acetic acid and 5-(3,4-dihydroxyphenyl)-gamma-valerolactone as the major metabolites. J Agric Food Chem. 2009;57:1084-92.

89. Crozier A, Del Rio D, Clifford MN. Bioavailability of dietary flavonoids and phenolic compounds. Mol Asp Med. 2010;31:446-67.

90. Monagas M, Urpi-Sarda M, Sanchez-Patan F, Llorach R, Garrido I, GomezCordoves $C$, et al. Insights into the metabolism and microbial biotransformation of dietary flavan-3-ols and the bioactivity of their metabolites. Food Funct. 2010;1:233-53.

91. Cornish HH, Christman A. A study of the metabolism of theobromine, theophylline, and caffeine in man. J Biol Chem. 1957;228:315-23.

92. Damm I, Enger E, Chrubasik-Hausmann S, Schieber A, Zimmermann BF. Fast and comprehensive analysis of secondary metabolites in cocoa products using ultra high-performance liquid chromatography directly after pressurized liquid extraction. J Sep Sci. 2016;39:3113-22.

93. Jang S, Sun J, Chen P, Lakshman S, Molokin A, Harnly JM, et al. Flavanol-enriched cocoa powder alters the intestinal microbiota, tissue and fluid metabolite profiles, and intestinal gene expression in pigs. J Nutr. 2016;146:673-80.

94. Langer S, Marshall LJ, Day AJ, Morgan MRA. Flavanols and methylxanthines in commercially available dark chocolate: a study of the correlation with nonfat cocoa solids. J Agric Food Chem. 2011;59:8435-41.

95. Garcia-Aloy M, Rabassa M, Casas-Agustench P, Hidalgo-Liberona N, Llorach R, Andres-Lacueva C. Novel strategies for improving dietary exposure assessment: multiple-data fusion is a more accurate measure than the traditional singlebiomarker approach. Trends Food Sci Technol. 2017;69:220-9.

96. Scientific Committee on Food EC, Health and Consumer Protection Directorate General. : Opinion of the Scientific Committee on Food on glycyrrhizic acid and its ammonium salt. 2003. https://ec.europa.eu/food/ sites/food/files/safety/docs/sci-com_scf_out186_en.pdf (Accessed 8 June 2018), 10 April 2003.

97. Vaya J, Belinky PA, Aviram M. Antioxidant constituents from licorice roots: isolation, structure elucidation and antioxidative capacity toward LDL oxidation. Free Radic Biol Med. 1997;23:302-13.

98. FoodComEx. 2017. http://foodcomex.org/. Accessed 11 June 2017.

Ready to submit your research? Choose BMC and benefit from:

- fast, convenient online submission

- thorough peer review by experienced researchers in your field

- rapid publication on acceptance

- support for research data, including large and complex data types

- gold Open Access which fosters wider collaboration and increased citations

- maximum visibility for your research: over $100 \mathrm{M}$ website views per year

At BMC, research is always in progress.

Learn more biomedcentral.com/submissions 